\title{
Trip Generation by Transportation Mode of Private School, Semi-private and Public. Case Study in Merida-Venezuela
}

\author{
Ángela María Quintero Petit and Mary Isabel Díaz Gallardo \\ Professor, University of the Andes, Venezuela \\ Emilio German Moreno González \\ Professor, Bolivarian Pontifical University of Bucaramanga, Colombia
}

\section{SUMMARY}

The trip generation model (TGM) is the first step in transportation forecasting, this is useful for estimating travel demand because it can predict travel from or to a particular land use. Typically, the analysis focuses in residential trip generation as a function of the social and economic attributes of households, but nonresidential land use suggests others variables. Travel generator poles such as: Private school, Semi-private and Public, have not been studied in Venezuela. The TGMs that shows the Institute of Transportation Engineers (ITE), EE.UU, are used typically and could be not appropriate. By using stepwise regression and transformation of data, high correlation coefficients and substantial improvements in the variability of data from several schools they were found. The trip generation rates (TGRs) by transportation mode: walking, motorcycle, public transport and cars, can be compared and be included in the Ibero-American Network of travel attractors poles.

\section{INTRODUCTION}

The TGRs allow prediction of land-use behavior, specifically in a place with uses of similar characteristics, i.e., allows foreseeing the impact on adjacent street prior to occupation land and to implement measures that mitigate potential impacts. TGMs allow determining specific accessibility requirements, estimating the future traffic intensity, and supporting proposals of improvements for transportation systems. Among the major limitations found in Venezuela and Colombia to make these forecasts is the lack of data for an adequate estimation. As a result, the rates of the Institute of Transportation Engineers (ITE), based on non-local data, are widely used. On the other hand, local adaptation sometimes requires the acquisition of data on existing structures with similar characteristics, which represents great cost. The Trip Generation Handbook (2001) recommends studies of local TGRs, for this reason, the University of the Andes (Venezuela) in conjunction with Bolivarian Pontifical University (Colombia), they have initiated study to obtain TGRs to different land uses. A greater effort is required to consolidate a wide database applicable to local environment and that spans to a large number of land uses and cities. The results show models that can be used to estimate traffic intensity by transportation mode applicable to different schools. Thus, TGRs relative to 32 schools were determinate; 11 private, 10 semi-private, and 11 
public schools, all located in Mérida-Venezuela. Surveys were applied to samples of the population in each school, which yielded different models according to the variables used by the ITE. Dataset allows determining the trip intensity generated by each transportation mode according to its distribution percentage. The results could be used to predict trips by school in different places and similar cities, an effort to add new land uses should be made in future work.

\section{LITERATURE REVIEW}

It is gives continuity to study of Quintero et al. (2014) who obtained TGRs and TGMs for trips estimation in private and semi-private schools, where the models depend of the students and employees. For data collection, surveys were applied to a sample of 22 schools and the modal distribution for private and semi-private schools showed that the most used mode was the car followed by public transport. The rates were obtained with the use of total trips by modes such as cars, school vans and taxis, and the TGMs were obtained by step-wise regression and Box-Cox optimization in private school case, where students plus employed was explicative variable, while for semi-private schools was only students. Grando et al. (2014) analyzed mobility on the Federal University in Brazil; they carried out a diagnosis of current situation with regard to transportation modes and entry-exit of the campus. Work was based on origin-destination surveys, where it characterizes the population and the arrival-departure trips. The study showed that the trips were done $56 \%$ in car and $23 \%$ on public transport, followed by walking and cycling mode. They found low efficiency and poor planning of public transport which stimulates car use and motorcycle, and they say that quantify the trips is useful to support improve measures of infrastructure around to the study center and to ensure short-term mobility.

The low quality of service provided by public transport on Federal University of Pernambuco motivated a study to evaluate aspects of mobility (Meira et al., 2014). The campus has 35,000 people who are mobilized by 14 bus lines and generate more than 40,000 trips/day, which represent an important generator pole. They found a strong prevalence of public transport but an excessive transfers and high travel-times influence academic performance. The data collection was done with 1000 interviews/day and proportionality among users. The used data was upon: center to which belongs; user (student, professor, worker, etc.); income (based on minimum salary); origin time, arrival and scheduled departure time; origin place; and used mode (in car was asked about use of parking and how many people traveled; in public transport, how many transfers were made). The results showed that $60 \%$ used public transport and $30 \%$ car and the average travel-time of public transport was above of the average for all modes $(1.07 \mathrm{~h})$. Also, users complain about the lack of direct bus lines, i.e., there is concern about excessive transfers.

Souza and Rodrigues (2015) present TGMs obtained for residential buildings in Metropolitan Area of Belo Horizonte, through survey in a typical week, in hours from 6 to 
10 am and 4 to $8 \mathrm{pm}$. The cars and motorcycles were quantified without taking into account the vehicle occupation, and people who were walking, were questioned about if they arrived walk or in public transport for the mode segregation. Two models were found; in morning peak hour and evening peak. Through comparison of trips sampling with trips by models; an absolute error of $35 \%$ in the morning and $10 \%$ in the evening was found, i.e., the model estimates trips less than real value which suggest an adjusted factor of 1.35 and 1.10, respectively. Bartlett (n. d.) designates that the estimation come from of generator poles with similar characteristics to those whose impact it want provide; but TGRs are the movements generated divided by the activity size during specific period of time. For these reason, countries as: USA, United Kingdom, Australia, Brazil, and others, work in proper rates based on observation. The ITE data show that rates can vary with activity size, so a larger Mall can attract less travel than other smaller; also, the definition of independent variable can vary, i.e., if is area, can be considered total built or effectively used. These problems can be avoided by mean of standardizing of land-use definitions. The rates are constant for different geographic areas, over time, regardless of year season and/or income level and for different cultures. Obviously there are weaknesses but they can be widely overcome with the selection of appropriate rates. The used rates to assess trips must come from of similar culture, land use, activity size and economic development. This latter point motivates to work in reach local rates that could be used to make appropriate estimations, avoiding choose those with different geographic and cultural realities.

\section{METHODOLOGY}

The data was obtained through surveys to a sample of 32 schools. The results correspond to dataset taken between 2011 and 2015, and only taking into account the students and employees as variables. Students include pre-school level, basic and middle, and the employees include teachers, administrative staff and worker. The caption was applied in both principal variables and the survey includes specific questions. See Figure 1.

1. How do you reach the school?

a. Private car with a relative, neighbor or friend

b. School van

c. Taxi

d. Public transport

e. Walk

f. Driving own vehicle

g. Motorcycle

2. When you arrive in private car, taxi, bus or motorcycle, there is more people arriving to same school in the same vehicle. How many?

Fig. 1 - Survey applied for arrival period and departure

The questions were formulated in order to quantify trips made by people with a same origin- 
destination pattern and in the same vehicle, trying avoiding data redundancy. In the initial phase of sampling the surveys were applied to private schools and the motorcycle was not a clear mode choice, but, given the increased use of this mode was applied in semi-private and public schools. The survey was directly delivered for respective filling, while smaller students with difficulty in filling format, were grouped for the oral questions formulation. Interviewer in this case registered responses from raised hands and the teacher was key for a good results. The sampling contents $60 \%$ of the population in each variable, and was selected through random numbers. Some data through vehicle counts are taken in access to school, at entry and exit hours. This technique allowed caption the trips in car, school van, taxi, motorcycle and walking; the trips on public transport had disadvantage, because bus stop was far of counts area and could has been quantified as walking. The results obtained for sampling were expanded to the population and data was grouped to private, semi-private and public schools. Expansion was made through equation 1.

$$
T_{i}=\frac{t_{i} \cdot P}{m}
$$

$\mathrm{T}_{\mathrm{i}}$ : expected trips by mode $\mathrm{i}$, full students and employees of the school

$\mathrm{t}_{\mathrm{i}}$ : number of trips by mode $\mathrm{i}$, of the students and employees sample

P: population, students and employees of the school

$\mathrm{m}$ : sample size, students and employees of the school

The mode distributions were used in order to make comparisons and determine a model by mode. Each TGR were calculated by the ratio between total trips by mode and independent variable; where the students and employees were more reliable parameters. The TGR was obtained for each mode on each school by equation 2 . The pondered averages by independent variable with its respective statistical analysis were calculated separately for private, semiprivate and public schools. See equation 3.

$$
T G R_{i}=\frac{T_{i}}{I V}
$$

TGR $\mathrm{i}$ : trip generation rate by model i for school

IV: independent variable associated with the school

$$
\overline{T G R}_{i-p o n d}=\frac{\sum_{j=1}^{n} T G R i . I V}{\sum I V}
$$

$\overline{T G R}_{i-\text { pond }}$ : pondered mean of trip generation rate to the mode $\mathrm{i}$

The total car trips that included school van and taxi, divided between the number of students 
and employees as indicated above determine TGRs, of this total excludes trips made by public transport. The TGRs are expressed in trips during peak hour, i.e., entry/exit to school. The rates for public transport, walking and by motorcycle mode were also obtained, but were considered of less vehicular impact. The trips in cars are represented by multiple regression and stepwise method that defined the more significant variables.

\section{RESULTS}

A way to validate the obtained rates was to apply appropriate statistical tests that allow inference. The atypical data was purified with special tests such as Grubbs' test and Dixon's test, based on approximate intensity to more reliable values with less influence of data that not correspond to a normal distribution. In this case there are few outliers that were purged to ensure a more consistent analysis. Satisfactory results were obtained that allowed visualizing the normal trend, despite the apparent variability, considering all most influential modes in street congestion around schools (Quintero et al., 2014). The study indicates that dataset can be used to the proposed prediction models that determine the trips generated by each school -private and semi-private-. The case of motorcycle mode and students in semiprivate school was out of the expected range as normal. However, for employees variable of this same school was within expected range. The students and employees were the best variables for private schools, while for semi-private school this corresponds to students. The semi-private school gets a very poor correlation when using employed variable. The multiple regression for private school implied Box-Cox optimization to reaching the best correlation, a multiple regression model is shown that describes relationship between Car trips (car, school van and taxi) and two independent variables. See Equation 4.

$$
\text { CarTrips }=\exp [1.475381 \cdot \operatorname{Ln}(0.084662 \cdot S+0.316643 \cdot E+0.885938)]
$$

CarTrips: number of trips in vehicle/hour

S: $\quad$ number of students

E: $\quad$ number of employees

The p-value in the ANOVA test is less than 0.05; there is a statistically significant relationship among variables (95\% of confidence level). The r-squared statistic indicates that model explains $99.02 \%$ of the variability and adjusted r-squared statistic, which is more suitable for comparing models with different independent variables, is $98.92 \%$. The standard error shows a standard deviation of 55.07. This value can be used to construct prediction limits for new observations. The mean absolute error (MAE) of 42.05 is the average value of the residuals. The Durbin-Watson statistic test determines if there is any significant correlation based on the order in which they occur. The highest p-value on the independent variables is 0.0226 , belonging to $\mathrm{E}$. Since the p-value is less than 0.05 , the term is statistically significant. The Equation 5 shows the fitted model for Semi-private Schools that best represent the data. A multiplicative model describes the relationship between Car Trips and 
Students. How p-value is less than 0.05 , there is a statistically significant relationship between Car Trips and S (95\%). The r-squared statistic indicates that the model explains $99.78 \%$ with correlation coefficient of 0.9989 . The standard deviation is 0.2788 and the MAE is 0.2226 .

$$
\text { CarTrips }=\exp [0.915109 \cdot \operatorname{Ln}(S)]
$$

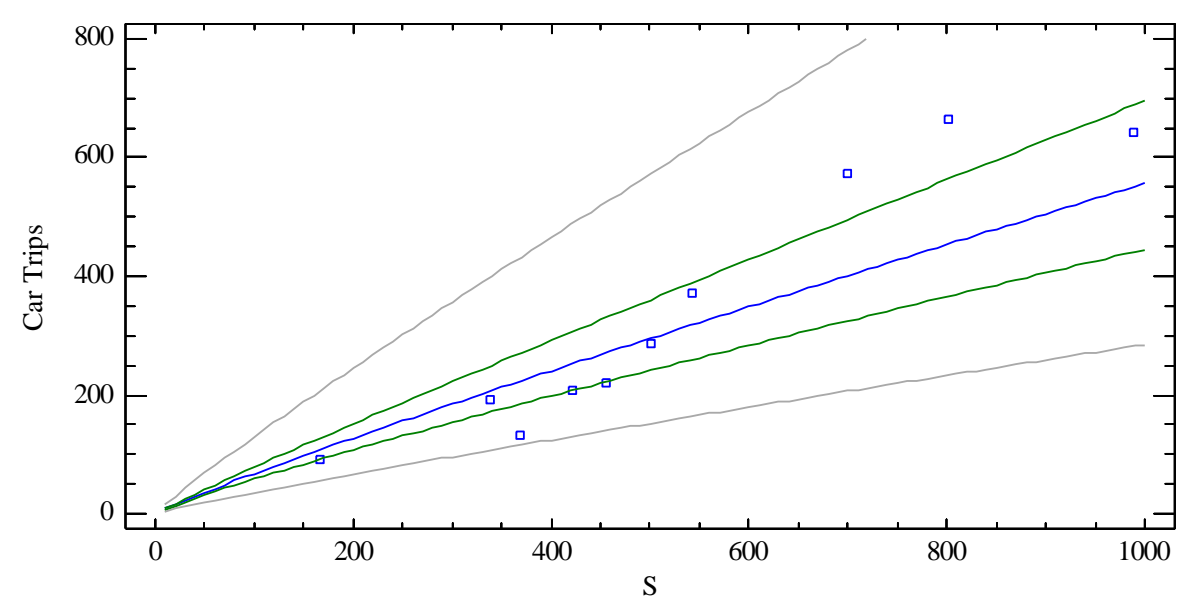

Fig. 2 - Car trips model for semi-private schools

The multiplicative model describes Car trips in function to Employees for Public Schools. See Equation 6. There is a statistically significant relationship among variables. The rsquared statistic is $97.98 \%$. The correlation coefficient is 0.9898 . The standard error is 0.6869 and the MAE is 0.493 . The Durbin-Watson test determines significant correlation.

$$
\text { CarTrips }=\exp [0.966184 \cdot \operatorname{Ln}(E)]
$$

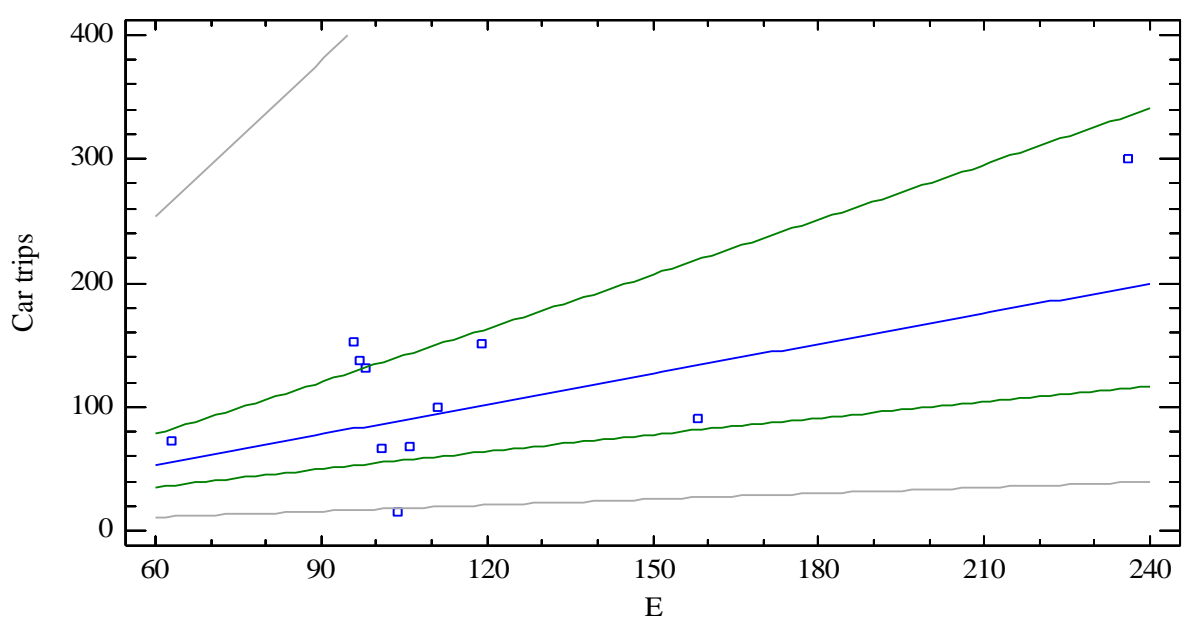

\section{Fig. 3 - Car trips model for public schools}

The trip generation models by motorcycle, walk and public transport are showed. Also, the trip-generation rates can be seen in Tables 1 and 2, respectively. 


\begin{tabular}{|l|l|c|}
\hline \multicolumn{1}{|c|}{ School description and mode } & \multicolumn{1}{|c|}{ TGMs } & $\begin{array}{c}\text { R-square } \\
\text { Adj. }(\%)\end{array}$ \\
\hline Private school (Motorcycle) & N/A & N/A \\
\hline Private school (Walk) & Walk trips $=\exp \left(0.615125^{*} \ln (\mathrm{S})\right)$ & 97.46 \\
\hline Private school (Public Transport) & PT trips $=\left(1.23346^{*} \ln (\mathrm{S})\right)^{\wedge} 2$ & 84.46 \\
\hline Semi-private school (Motorcycle) & Moto trips $=(0.158871 * \operatorname{sqrt}(\mathrm{S}))^{\wedge} 2$ & 95.28 \\
\hline Semi-private school (Walk) & Walk trips $=\exp \left(0.670492^{*} \ln (\mathrm{S})\right)$ & 98.85 \\
\hline Semi-private school (Pub. Transport) & PT trips $=\exp \left(1.2676^{*} \ln (\mathrm{E})\right)$ & 99.02 \\
\hline Public-school (Motorcycle) & Moto trips $=0.0942 * \mathrm{~S}-0.268355^{*} \mathrm{E}$ & 94.37 \\
\hline Public school (Walk) & Walk trips $=\exp (0.822687 * \ln (\mathrm{S}))$ & 99.81 \\
\hline Public school (Public Transport) & PT trips $=\exp \left(0.92512^{*} \ln (\mathrm{S})\right)$ & 99.93 \\
\hline
\end{tabular}

Table 1 - Summary of regression equations by mode and school

\begin{tabular}{|c|c|c|c|c|c|c|c|c|c|c|c|c|}
\hline & \multicolumn{12}{|c|}{ TGRs } \\
\hline & \multicolumn{4}{|c|}{ Private } & \multicolumn{4}{|c|}{ Semi-Private } & \multicolumn{4}{|c|}{ Public } \\
\hline & $\mathrm{C}$ & $\mathrm{M}$ & $\mathrm{W}$ & PT & $\mathrm{C}$ & $\mathrm{M}$ & $\mathrm{W}$ & PT & $\mathrm{C}$ & $\mathrm{M}$ & $\mathrm{W}$ & PT \\
\hline PMR* & 0.77 & N/A & 0.10 & 0.13 & 0.58 & 0.02 & 0.12 & 0.27 & 0.16 & 0.04 & 0.27 & 0.53 \\
\hline $\mathrm{SD}^{* *}$ & 0.25 & N/A & 0.09 & 0.17 & 0.15 & 0.01 & 0.07 & 0.13 & 0.08 & 0.02 & 0.07 & 0.09 \\
\hline $\mathrm{VC}^{* * * *}$ & 0.32 & N/A & 0.90 & 1.33 & 0.26 & 0.43 & 0.56 & 0.47 & 0.51 & 0.46 & 0.26 & 0.17 \\
\hline
\end{tabular}

*PMR: pondered mean rate; **SD: standard deviation; ***VC: variability coefficient

Table 2 - Summary of TGRs by mode and school

\section{CONCLUSSIONS}

The trip generation rates for private, semi-private and public schools are useful based on the best variables. These rates could be applied to the different mode, but the car (particular vehicle, school van and taxi) is who most impact has on adjacent streets to the schools. The TGRs adjusted to a normal distribution and the rates with more reliability were: Cars in private and semi-private schools and Public Transport and Walking for public schools.

The stepwise regression allows obtained best trip models per hour per school; in private schools depended of both variable (students and employees), while for semi-private schools and public were the students, and employees, respectively. Each result shows strong correlation according to the results. By mean of data transformation for private schools, i.e., Box-Cox optimization makes residuals more normal and less heteroskedastic, in Employees variable specifically. This optimization reaches normality and best linear relationship among variables.

These results could be extrapolated to similar places to Mérida, cities like BucaramangaColombia for example, as a second step to achieve models more generalized. The performance of the models should be analyzed under different scenarios to evaluate its 
robustness and reasonability. Only in this way can be used to simulate the impact, as support of diverse policies that can be applied in a future.

\section{ACKNOLEDGEMENTS}

The research is part of the program that leads the University of the Andes (Venezuela) in conjunction with the research Group in Transport, Infrastructure and Territory -GITIT- of the Bolivarian Pontifical University (Bucaramanga, Colombia).

\section{REFERENCES}

BARTLETT, R. (n.d.). Internationality of trip rates.

GRANDO GOLDNER, L., FORTUNATO MARCON, A., IZZI, A. and GIARETTA, R. (2014). Diagnóstico da Mobilidade em um Campus Universitário: o Caso da UFSCTrindade.

ITE - INSTITUTE OF TRANSPORTATION ENGINEERS (2001). Trip Generation Handbook - An ITE Recommended Practice. $6^{\text {a }}$ Ed. Washington D.C

MEIRA, L., ALVES MAIA, M., BRASILEIRO, A. and OLIVEIRA DE ANDRADE, M. (2014). A influência da qualidade do transporte público na rotina acadêmica: $O$ caso da Universidade Federal de Pernambuco.

QUINTERO, P., DÍAZ, G. and MORENO, G. (2014). Travel Estimation Model Generated by Public and Private Schools, According to Different Transport Modes. Case Study MéridaVenezuela. Procedia - Social and Behavioral Sciences, 160, pp.509-518.

SOUZA DE OLIVEIRA, P. and RODRIGUES, F. (2015). Calibração de modelo de geração de viagens para condomínios de edifícios residenciais. 